\title{
Determinants of active commuting
}

\author{
Bengt Kayser \\ University of Geneva, Faculty of Medicine, EEPS/ISMMS, Geneva 1205, Switzerland
}

Available online 20 September 2007

In this issue of Preventive Medicine Bringolf-Isler et al. (Bringolf-Isler et al., 2008) report on factors associated with active commuting to school in more than 1000 children aged 6-14 years in a German-speaking, a French-speaking, and a bilingual area in Switzerland. This study design naturally allows for separating cultural from other determinants of selftransportation. Switzerland may be a small country with a socio-economically homogenous population but it nevertheless has different language regions with subtle differences in culture.

Active commuting to school of children is still the rule in Switzerland (on average $78 \%$ in this study), which is high compared to some other countries, and probably reflects a high perceived safety by parents of the way to school $(63 \%$ of parents judged the way to school sufficiently safe).

But, as in other countries, childhood obesity is on the rise in Switzerland and there are indications that active commuting to school is decreasing. A better understanding of the factors related to active commuting is needed to keep the high prevalence of active transportation to school. Using a geographical information system (GIS) the authors identified distance to school and density of major traffic hurdles as important factors. They also found that belonging to the French-speaking population is related to less active modes of commuting as compared to the German-speaking population. The authors recommend that, in order to keep active commuting levels high, the route to school should remain relatively short and safe. They also underline the importance of taking cultural differences into account, as exemplified by the differences found between German- and French-speaking sub-populations, even though the origin of the cultural effect remains poorly understood (Stamm and Lamprecht, 2005). Limiting factors of the study are its crosssectional design precluding conclusions on causality and the lack of objective measures of physical activity levels (the study used parental questionnaires).

E-mail address: bengt.kayser@medecine.unige.ch.
Lack of physical activity during childhood is an important health risk factor. Because of the developing pandemic of sedentarity-related disorders, promotion of physical activity is a public health priority (World Health Assembly, 2004). The regulation of habitual physical activity is complex. One important determinant appears to be the built environment. A recent US report (Committee on Physical Activity, 2005) insisted that "research on the relationship between the built environment and physical activity is at a pivotal stage" and that "policy prescriptions require a better understanding of causal connections than currently exists, as well as of the strength of these connections and their impact on population subgroups." The report strongly urged for more research and funding in this field; interdisciplinary and international research bringing together epidemiology, urbanism, architecture, geography and sociology, using technology like GIS, global positioning systems, pedometers, accelerometers and the like. The Bringold-Isler article (Bringolf-Isler et al., 2007-this issue) is a nice example of such research and provides much needed data for policy makers. Making physical activity the norm by rendering sedentary behavior more difficult is necessary to relieve the global burden of sedentarity-related disorders. Much remains to be researched and a major challenge subsists: to design and conduct more interventional studies which can provide evidence for best public health practices to promote walking and cycling.

\section{References}

Bringolf-Isler, B., Grize1, L., Mäder, U., Ruch, N., Sennhauser, F., BraunFahrländer, C., SCARPOL team, 2008. Personal and environmental factors associated with active commuting to school in Switzerland. Prev Med. 46, $67-73$.

Committee on Physical Activity, Health, Transportation, and Land Use of the Transportation Research Board and the Institute of Medicine of the National academies, 2005. Does the built environment influence physical activity? Examining the evidence. Transportation Research Board Special Report 282, Washington, National Academy of Sciences.

Stamm, H.-P., Lamprecht, M., 2005. Structural and cultural factors influencing physical activity in Switzerland. J. Public Health 13, 203-211.

World Health Assembly, 2004. Global Strategy on Diet, Physical Activity and Health. WHO, Geneva. 